\title{
Correct anteroposterior patterning of the zebrafish neurectoderm in the absence of the early dorsal organizer
}

Máté Varga ${ }^{1,2^{*}}$, Shingo Maegawa ${ }^{1,3}$ and Eric S Weinberg ${ }^{1 *}$

\begin{abstract}
Background: The embryonic organizer (i.e., Spemann organizer) has a pivotal role in the establishment of the dorsoventral (DV) axis through the coordination of BMP signaling. However, as impaired organizer function also results in anterior and posterior truncations, it is of interest to determine if proper anteroposterior (AP) pattern can be obtained even in the absence of early organizer signaling.

Results: Using the ventralized, maternal effect ichabod (ich) mutant, and by inhibiting BMP signaling in ich embryos, we provide conclusive evidence that AP patterning is independent of the organizer in zebrafish, and is governed by TGF $\beta$, FGF, and Wnt signals emanating from the germ-ring. The expression patterns of neurectodermal markers in embryos with impaired BMP signaling show that the directionality of such signals is oriented along the animal-vegetal axis, which is essentially concordant with the AP axis. In addition, we find that in embryos inhibited in both Wnt and BMP signaling, the AP pattern of such markers is unchanged from that of the normal untreated embryo. These embryos develop radially organized trunk and head tissues, with an outer neurectodermal layer containing diffusely positioned neuronal precursors. Such organization is reflective of the presumed eumetazoan ancestor and might provide clues for the evolution of centralization in the nervous system.

Conclusions: Using a zebrafish mutant deficient in the induction of the embryonic organizer, we demonstrate that the AP patterning of the neuroectoderm during gastrulation is independent of DV patterning. Our results provide further support for Nieuwkoop's "two step model" of embryonic induction. We also show that the zebrafish embryo can form a radial diffuse neural sheath in the absence of both BMP signaling and the early organizer.
\end{abstract}

\section{Background}

The body plan of developing animal embryos is initially generated by establishment of the anteroposterior (AP) and dorsoventral (DV) axes. The dorsal organizer (i.e., Spemann organizer and homologous structures) is clearly important in formation of the DV axis (reviewed in [1-3]), but its role in AP axis development has been controversial (reviewed in $[4,5]$ ). Failure to form the Spemann organizer in frogs and fish [6-8] results not only in the absence of dorsal tissues, but also in the loss of anterior regions of the embryo. Nevertheless, there is also evidence that at least some degree of proper AP patterning occurs with surgical removal or genetic

\footnotetext{
* Correspondence: m.varga@ucl.ac.uk; eweinber@sas.upenn.edu 'Department of Biology, University of Pennsylvania, Philadelphia, PA 19104, USA

Full list of author information is available at the end of the article
}

ablation of the organizer in mouse, chick, and zebrafish embryos [9-17]. In zebrafish and Xenopus embryos unable to form a dorsal organizer, head neurectodermal markers are still expressed in proper relative AP order if BMP signaling is absent $[8,18,19]$. The orientation of the AP axis with respect to the animal/vegetal (AnVeg) axis has also been disputed. Some have argued for the equivalence of the AP axis with the "classic" DV axis of anamniotes [20-22], while others have proposed a concordance of the AP and AnVeg axes in these groups [5]. As the function of the organizer may obscure an underlying mechanism that establishes AP pattern, we chose to further study the control of AP axis formation in embryos genetically blocked in the ability to form a dorsal organizing center.

Embryos bred from females homozygous for the ich mutation (ich embryos) show a reduction of maternal $\beta$ -
C Biomed Central

C 2011 Varga et al; licensee BioMed Central Ltd. This is an Open Access article distributed under the terms of the Creative Commons Attribution License (http://creativecommons.org/licenses/by/2.0), which permits unrestricted use, distribution, and reproduction in any medium, provided the original work is properly cited. 
catenin-2 expression. Furthermore, treatment of wildtype embryos with a morpholino antisense oligonucleotide (MO) targeting $\beta$-catenin- 2 causes a failure of organizer formation and loss of anterior tissues, whereas loss of $\beta$-catenin- 1 alone has no ventralizing or posteriorizing effects [8]. Using a TOP-GFP Wnt-reporter line, we showed that while the MO against $\beta$-catenin-2 ( $\beta$ cat2MO) could eliminate the dorsal marginal expression of the transgene in shield stage embryos, a MO against $\beta$-catenin-1 ( $\beta$ cat $1 \mathrm{MO})$ had no effect in this region [23]. In contrast, the germ-ring transgene expression was abolished only when the two MOs were administered together, showing that this domain of expression was mediated redundantly by both $\beta$-catenins [23]. Injection of both MOs into wild-type embryos, or of $\beta$ cat1MO to ich embryos already deficient in $\beta$-catenin-2 expression, caused the ectopic induction of chordin (chd) and noggin1 (nog1) around the blastodermal margin of the embryo $[8,23]$. Such embryos deficient in both $\beta$-catenins develop a distinctive phenotype at 24 hpf (termed 'ciuffo'), in which a protrusion of tissue from the vegetal end of the yolk expresses neurectodermal markers in an apparently proper AP pattern. This expression is dependent on chd [8,23]. The massive expression of chd in $\beta$ cat $1 \mathrm{MO}+\beta$ cat $2 \mathrm{MO}$-treated embryos would be expected to result in a marked inhibition of BMP signaling due to the direct binding of Chd to BMP ligand [24].

In the work presented here, we first show that $i c h$ embryos injected with $\beta$ cat $1 \mathrm{MO}+\beta$ cat $2 \mathrm{MO}$ or with bmp2bMO alone both exhibit loss of BMP signaling and upregulate chd. Previously, we demonstrated that in 'ciuffo' embryos, key specific markers of the early organizer are never induced [8] and expression of goosecoid (gsc) [25] and chd are dependent on the endogenous, germ-ring expression of Nodal homologues [23].

Here we provide definitive evidence that the upregulation of chd in bmp2bMO-treated ich embryos is not due to the ectopic induction of organizer tissue, since other typical markers of the early and late dorsal signaling center are not induced. The ectopic expression of chordin appears to be a consequence of downregulation of BMP signaling, and not due to radialization of an early dorsal organizing center. Using embryos at several developmental stages, we show that both anterior and posterior neurectodermal markers are expressed with correct AP pattern, even in the absence of the organizer, as long as BMP signaling is inhibited. We then show that the same pathways that are involved in setting up the AP neurectodermal pattern in wild-type embryos Wnt-, Nodal-, and FGF signaling - are required for elaboration of the full AP pattern in the absence of BMP and organizer signals. Finally, we examined the morphology of the neurectodermal tissue in 'ciuffo' embryos and found that cells with neuronal identity are organized in a sheath of mesoderm and endoderm, similar to the neural net present in cnidarians. We speculate that during the evolution of bilaterian precursors, the establishment of a DV-oriented BMP signaling gradient during embryogenesis resulted in the transformation of an outer radially organized, AP-patterned neural sheath, into the stereotypical vertebrate neural tube. This view extends the recent analysis of Meinhardt [5], which regards the generation of AP pattern of the vertebrate brain as an organizer-independent, ancestral, radially symmetric system.

\section{Results}

Embryos deficient in canonical Wnt signaling show loss of BMP signaling

To better understand the effect of inhibition of Wnt/ $\beta$ catenin signaling on formation and patterning of neurectoderm in the zebrafish embryo, we examined to what degree BMP signaling was affected by the elimination of expression of the two $\beta$-catenins by administration of $\beta$ cat $1 \mathrm{MO}+\beta$ cat $2 \mathrm{MO}$ to ich embryos. As we had previously shown that such inhibition results in a high level of ectopic chd expression, we expected to find a very low level of BMP signaling in these embryos. We were also interested in whether the resulting inhibition of BMP signaling was equivalent to direct inhibition of BMP expression attained by injection of an $\mathrm{MO}$ against bmp $2 b$ (bmp2bMO) [26]. The degree of BMP signaling in these embryos was visualized by examining the distribution of phosphorylated Smad1/5 (P-Smad5), an indicator of cells actively transducing BMP signaling $[27,28]$. Administration of the two $\beta$-catenin MOs to ich embryos was in fact as effective as treatment of these embryos with bmp2bMO in eliminating BMP signaling in all regions except at the very animal pole (Figure 1). Wild-type embryos at 50\% epiboly exhibit a gradient of nuclear P-Smad5 with the most intense staining at the ventral-most area of the embryo, and exclusion of $\mathrm{P}$ Smad5 from the dorsal side (Figure 1A; [29]) where chd is expressed (Figure 1F). ich embryos show an expansion of P-Smad5 throughout the embryo, with no evidence of an activity gradient (Figure $1 \mathrm{~B}$ ) or of chd expression (Figure 1G). Injection of bmp2bMO into ich embryos at a concentration which phenocopies the swirl (swr) mutation in wild-type embryos [30,31], eliminates PSmad5 in the embryo (Figure $1 \mathrm{C}$ ), and results in a massive expression of chd (Figure $1 \mathrm{H})$. We observe a similar phenotype when large amount of chd mRNA is injected into ich embryos (not shown). The loss of Bmp2b activity is known to impair expression of bmp 4 and $b m p 7$ $[32,33]$; thus, it is not unexpected that global BMP-signaling is lost in the bmp2bMO-treated embryos. Injection of $i c h$ embryos with $\beta$ cat $1 \mathrm{MO}+\beta$ cat $2 \mathrm{MO}$ has very 


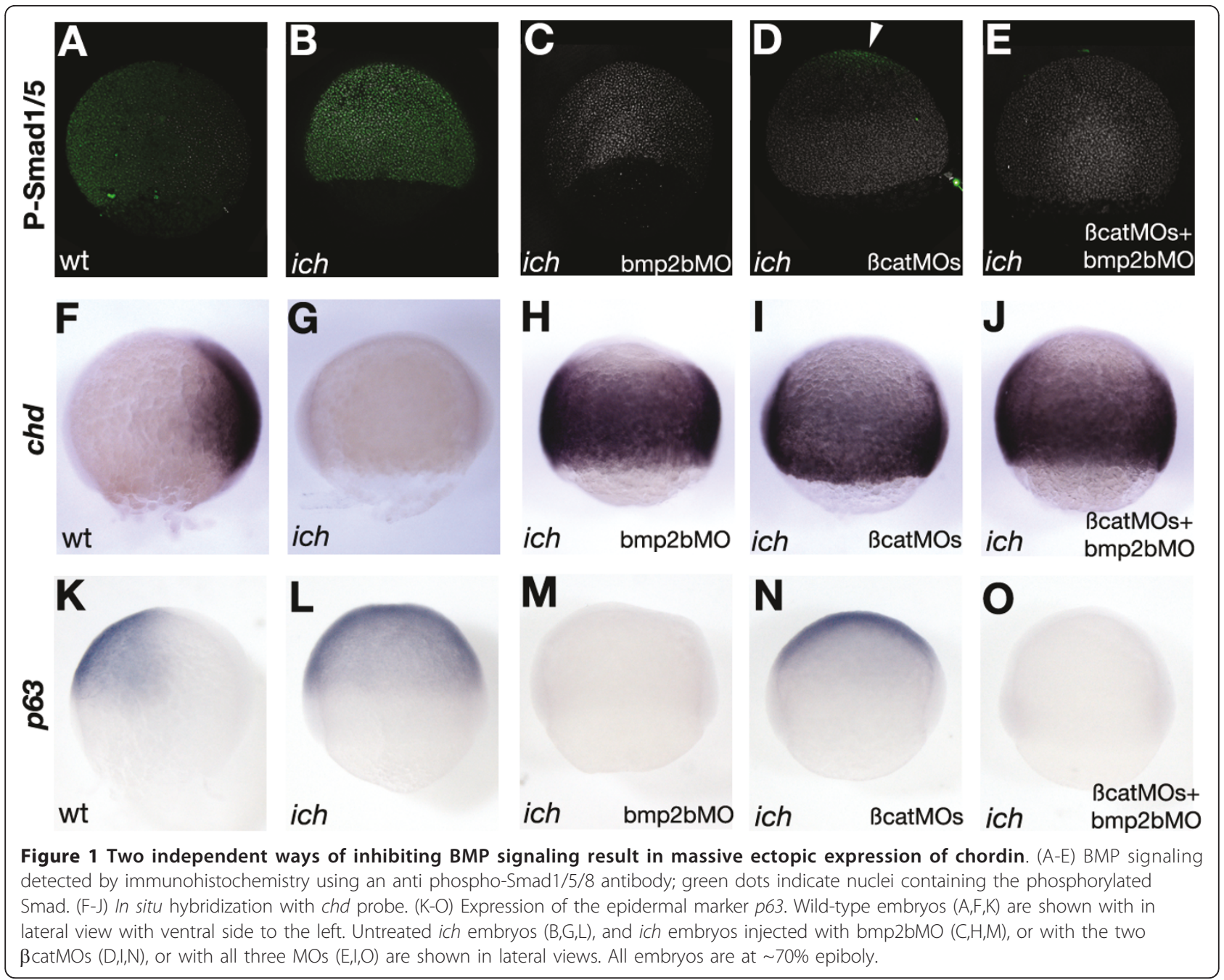

much the same effect on P-Smad5 (Figure 1D) and chd expression (Figure 1I) as bmp2bMO injection, except that nuclear P-Smad5 can be seen at the animal pole (Figure 1D arrowhead). Injection with the three MOs results in embryos with a distribution of P-Smad5 and chd expression very much as in embryos injected with bmp2bMO alone.

Ectopic expression of chd is also correlated with the repression of epidermal markers, as posited by the "neural default model" [34]. The ubiqitous expression of the epidermal marker $p 63$ [35] observed in ich embryos (Figure 1L) is reduced upon the injection $\beta$ catMOs (Figure $1 \mathrm{~N}$ ), and completely abolished when bmp2bMO is used alone or in combination with the other MOs (Figure $1 \mathrm{M}, \mathrm{O})$. This latter result shows that in the absence of BMP signaling ectodermal cells can not acquire epidermal fates.

In summary, two different methods - injection of $i c h$ embryos with bmp $2 \mathrm{bMO}$ or with $\beta$ cat $1 \mathrm{MO}+\beta$ cat $2 \mathrm{MO}$ (i.e., 'ciuffo' embryos) - can be utilized to obtain embryos lacking the organizer and BMP signaling.

\section{Impairment of BMP-signaling does not result in ectopic organizer formation}

One important question to address is whether the induction of chd in MO-treated ich embryos reflects the ectopic induction of organizer tissue, or it is a transcriptional consequence of global BMP signaling downregulation. Our previous results showed that in 'ciuffo' embryos one of the earliest markers of endogenous organizer induction, bozozok/dharma (boz) [36-38] is never expressed, and the circumferential, germ-ring expression of later dorsal markers such as gsc and chd is induced with a significant delay (at 50\% epiboly instead of at $30 \%$ epiboly) compared to their dorsal appearance in wild type embryos $[8,23]$. We now examined the expression of these markers in bmp2bMO-injected embryos (Figure 2). In contrast to chd (Figure 2I), 

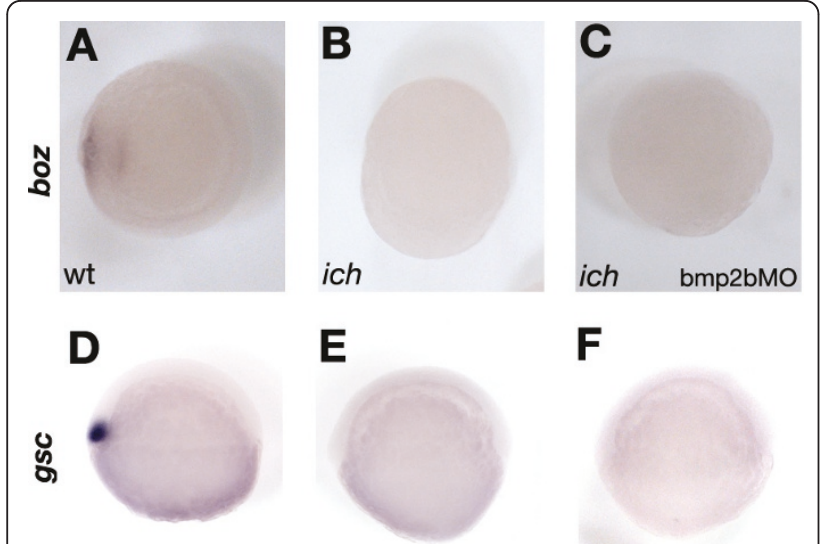

wt

ich

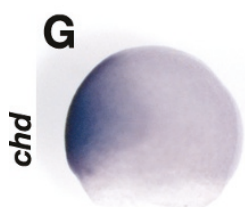

wt

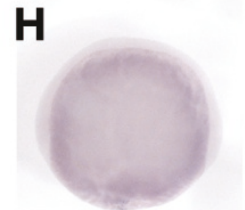

ich
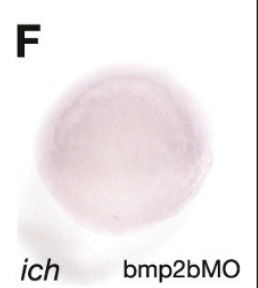

I

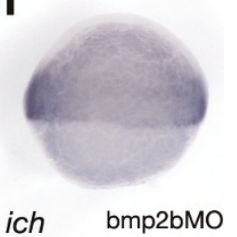

Figure 2 Inhibition of BMP signaling in ich embryos does not result in organizer induction. In situ hybridization with boz $(A, B, C)$, gsc $(\mathrm{D}, \mathrm{E}, \mathrm{F})$ and chd $(\mathrm{G}, \mathrm{H}, \mathrm{I})$ probes in uninjected ich and bmp2bMOinjected ich embryos demonstrate that while impaired BMP signaling results in the transcriptional derepression of chd at the margins of the embryo $(H, I)$, other characteristic organizer genes such as boz and gsc are not induced (C and F). (In A-C dome staged embryos are shown from an animal pole view, D-F and G-I present lateral views of 50\% epiboly and 70\% epiboly staged embryos, respectively. For wild-type embryos, dorsal is on the left.)

expression of boz (Figure 2C) and gsc (Figure 2F) was not detected in such embryos.

Unlike some other components of the early DV transcriptional network (e.g. squint (sqt) [15] and $f g f 8$ [39]) that are first expressed dorsally at sphere stage and then followed by a pan-germ-ring upregulation at $~ 30 \%$ epiboly, $b o z$ and $g s c$ are exclusively expressed in the dorsal side of developing wild type embryos, and are thus more definitive markers of the early dorsal signaling center and organizer. Furthermore, while the ectopic expression of these genes can induce a complete axis in zebrafish [40,41], the overexpression of chd alone cannot do so, neither in wild type [41], nor in ich embryos (our unpublished observations). As is the case also in Xenopus [42], coinjection of chd mRNA with a Wnt-antagonist can induce anterior neural structures and notochord (our unpublished observations). But, unlike Xenopus, where chd is found to be necessary for the induction of a complete secondary axis [43], in zebrafish chd is dispensable for $g s c$-induced secondary axis formation [41]. These results offer clear evidence that the ectopic expression of chd in bmp2bMO-injected ich embryos is due to a global de-repression of its transcription in the absence of BMP signaling [44], and is not the consequence of ectopic induction of organizer tissue.

\section{Inhibition of BMP signaling reveals normal AP neurectodermal patterning in the absence of the organizer}

At $10 \mathrm{hpf}$, cyp26, hoxb1b, otx 1 , and gbx1 clearly mark distinct neurectodermal territories in wild-type embryos: cyp 26 is expressed both in the anterior neurectoderm and in the most posterior region of the embryo (Figure $3 \mathrm{~A}, \mathrm{C}$, arrow and star, respectively) [45], hoxb1b marks neuroectoderm posterior to the prospective rhombomere 3/4 boundary (Figure 3B,C) [46]. In ich embryos, as expected [7], expression of otx1, hoxb1b, gbx1, and the anterior domain of cyp26, is absent (Figures 3D-F). Only the posterior domain of cyp26 is still expressed (Figure 3D), and its expansion is consistent with the observation that the most posterior neurectoderm still forms in ich embryos [47].

When injection of bmp2bMO is used to inhibit BMP signaling in these embryos, all four neurectodermal markers are expressed robustly in correct relative AP order in approximately the same AP position as in wild-type embryos (Figures 3G-I). The width of the expression domains is almost identical to those of wild-type embryos, but the expression is radial, extending completely around the embryo, rather than restricted to the dorsal side. (Similar results were observed with two other markers, otx 1 , expressed in the prospective forebrain and midbrain [48], and $g b x 1$, expressed from the midbrain/hindbrain boundary posterior towards rhombomere 2 [49] (Additional file 1, Figure S1A-I)).

When $\beta$ cat $1 \mathrm{MO}+\beta$ cat $2 \mathrm{MO}$ injection is used to inhibit BMP signaling, the results are similar in that all four markers are expressed in correct AP order (Figure 3J-L, Additional file 1, Figure S1J-L). The major difference is that expression is shifted towards the posterior of the embryo. The anterior cyp26 domain and the otx 1 and $g b x$ domains are wider, and the area of hoxb1b is restricted to a more posterior region of the embryo than is the case in wild-type or in bmp2bMO-injected ich embryos. We also tested the effects of injecting all three MOs on expression of the four markers (Figure 3M-O, Additional file 1, Figure S1M-O). Results for the more posterior markers $g b x 1$ and $h o x b 1 b$ are similar to the embryos treated with the two $\beta$ catMOs alone, while the expression of $o t x 1$ and the anterior domain of cyp 26 is expanded to encompass the whole anterior $60 \%$ of the neurectoderm. Treatment with bmp2bMO, but not with the two $\beta$ catMOs, eliminates BMP signaling in the animal pole region (Figure 1C-E), thus permitting expression of these anterior markers in the former, but not latter, embryos. The expansion of expression of the 


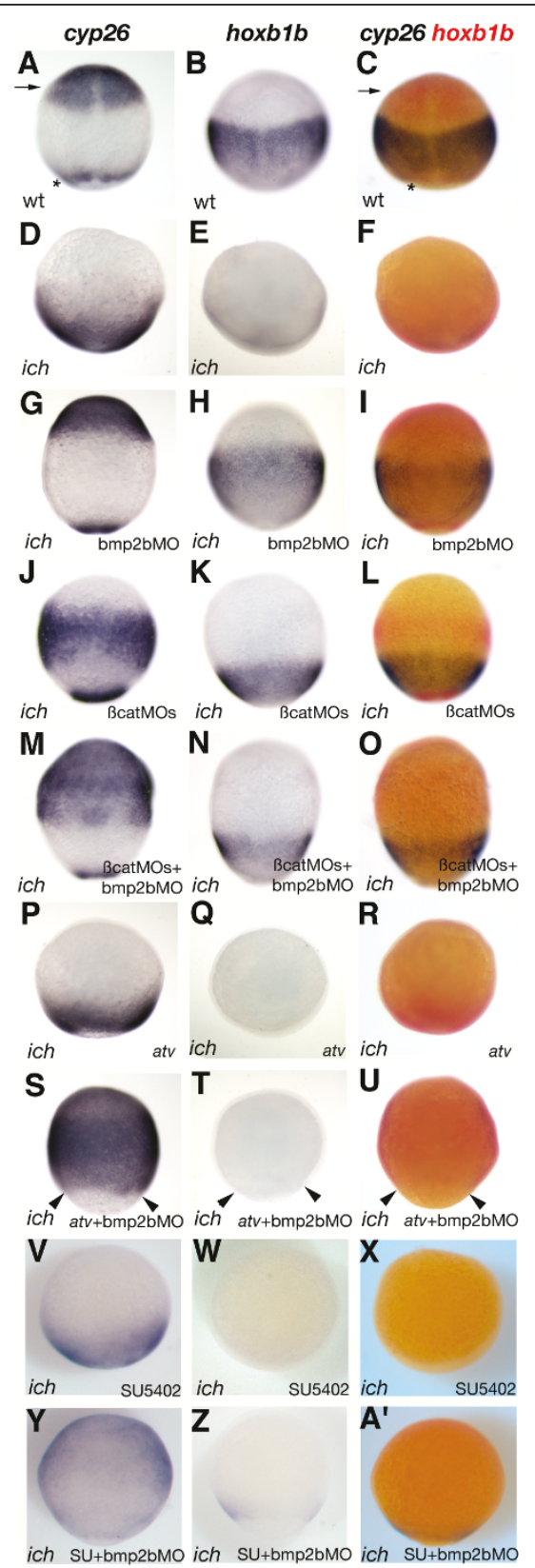

Figure 3 Embryos inhibited in both BMP signaling and organizer function form neuroectoderm with correct AP pattern at the end of gastrulation and this patterning can be modulated by similar mechanisms as in wild-type embryos. Single in situ hybridization with cyp26 $(\mathrm{A}, \mathrm{D}, \mathrm{G}, J, \mathrm{M}, \mathrm{P}, \mathrm{S}, \mathrm{V}, \mathrm{Y})$ or hoxb1b $(B, E, H, K, N Q, T, W, Z)$ probes or double in situ hybridization with both probes $\left(C, F, I, L, O, R, U, X, A^{\prime}\right)$ is shown for wild-type embryos (A-C), untreated ich embryos (D-F), or ich embryos treated with bmp2bMO $(\mathrm{G}-\mathrm{I})$, the two $\beta$-cat MOs $(J-\mathrm{L})$, bmp2bMO plus the two $\beta$-cat MOs $(\mathrm{M}-\mathrm{O})$, or ich embryos injected with antivin mRNA (P-R), antivin mRNA plus bmp2bMO (S-U), SU5402 (V-X), or SU5402 plus bmp2bMO (Y-A'). Wild-type embryos $[A-C]$ are shown in dorsal views, while ich embryos are shown in lateral views. The neuroectodermal and tailbud expression domains of cyp26 in wildtype embryos are marked with arrow and star, respectively. All embryos are at $\sim 100 \%$ epiboly. anterior markers toward the vegetal pole in the triple MO-treated embryos, compared to embryos treated with bmp2bMO alone, is evidence for a posteriorizing role of $W n t / \beta$-catenin signaling. When this signaling is eliminated by MO treatment, there is a marked posterior expansion of the zone of anterior identity.

Neurectodermal markers expressed at later times in these treated embryos also show correct relative AP patterning (Figure 4). At $11 \mathrm{hpf}$, the wild-type expression of both the eye-field marker $r \times 3$ (Figure 4A,C) [50] and the diencephalic marker $f k d 3$ (Figure $4 B, C$ ) [51] is completely absent in ich embryos (Figure 4D-F) but is conserved in the bmp2-morphant ich embryos (Figure 4GI). These results indicate that the AP patterning of postgastrula embryos is also independent of the presence of the organizer, as long as BMP signaling is absent. Identical results were obtained at this stage using the midbrain-hindbrain boundary (MHB) marker pax2.1 [52] and the hindbrain rhombomere 3 and 5 marker, krox 20 [53] (data not shown). The 11 hpf 'ciuffo' embryos also show correct relative AP patterning of $f k d 3$ (Figure $4 \mathrm{~K}$, L), pax2.1, and krox20 markers (data not shown). However, these embryos do not express $r \times 3$ (Figure $4 \mathrm{~J}, \mathrm{~L}$ ), the most anterior marker tested, presumably because of the active BMP signaling present at the animal pole.

A comparison of AP patterning in the neurectoderm of $\beta$ catMO-injected ich ('ciuffo') embryos and $b m p 2 b$ morphant embryos cannot easily be carried out at later stages. After $11 \mathrm{hpf}$, bmp2bMO-injected ich embryos, like $s w r / b m p 2 b$ embryos [30], begin to burst and die as a consequence of severe constriction movements. The 24 hpf $\beta$ cat1MO-injected ich embryos do survive and we already had good indication that their neurectoderm exhibited correct patterning $[8,23]$. Two-color in situ hybridizations with four probes (emx1, krox20, val, hoxb6b) showing distinct restricted expression along the AP axis confirm and extend these results (Additional file 2, Figure S2). While none of these markers were expressed in ich embryos (Additional file 2, Figure S2B, $\mathrm{E}, \mathrm{H})$, they were all expressed in correct relative spatial order in embryos co-injected with $\beta$ cat1MO and $\beta$ cat $2 \mathrm{MO}$. The more anterior markers (emx1 in Additional file 2, Figure S2A-C', krox20 in Additional file 2, Figure S2D-I') were detected proximal to the yolk, while the posterior markers (krox20 in Additional file 2, Figure S2A-C', val in Additional file 2, Figure S2D-F' and hoxb6b in Additional file 2, Figure S2G-I') were expressed distal to the yolk. These results, along with the data presented on $10 \mathrm{hpf}$ and $11 \mathrm{hpf}$ embyos, indicate that the patterning pathways necessary to establish major neuroectodermal territories (forebrain, hindbrain and neural tube) are functional in 'ciuffo' embryos, and that these regions are demarcated with correct relative AP pattern. 


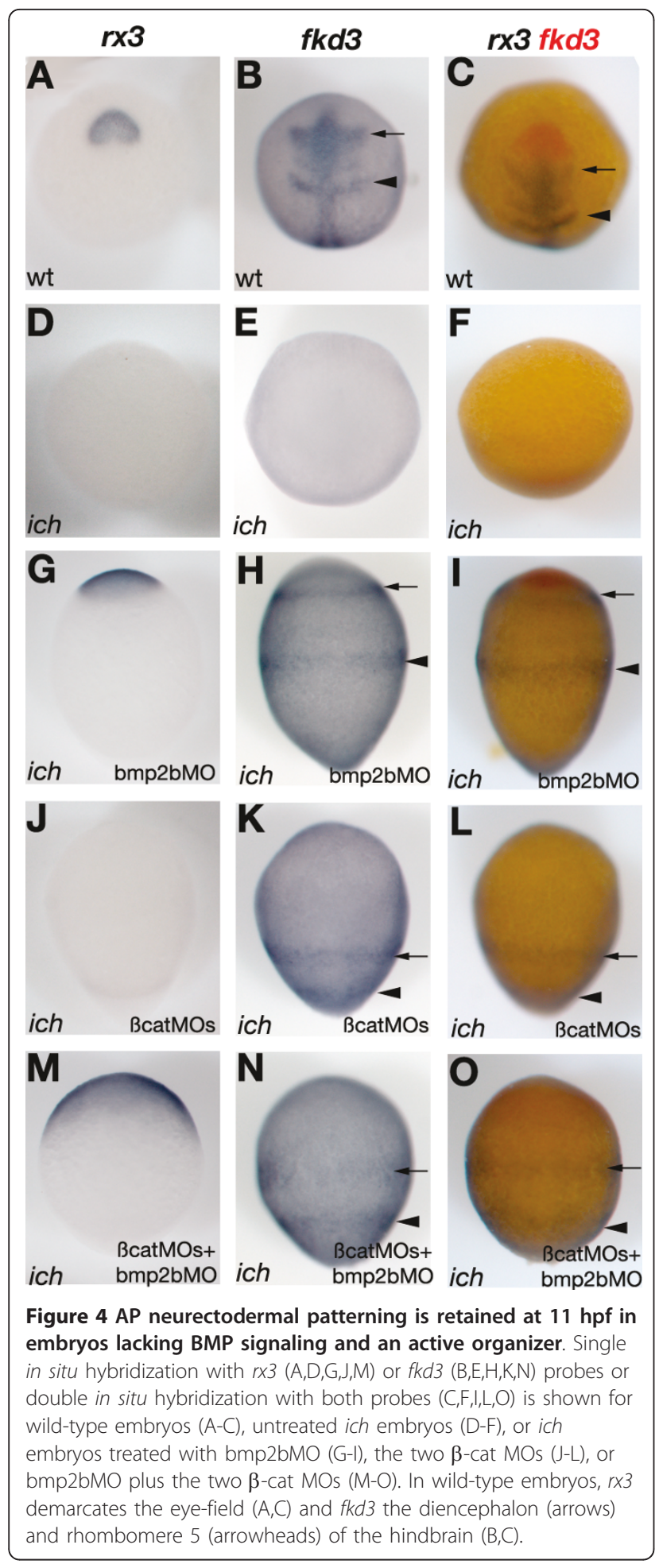

We also tested ich embryos injected with $\beta$ catMO1 or bmp2bMO for expression of the more posterior hox genes hoxb6b, hoxb8a, hoxa9a, hoxd12, and hoxc13a at 24 hpf. (The majority of bmp2bMO-injected embryos die during earlier stages of development, but 2\% survive to $24 \mathrm{hpf}$.) Although we were unable to obtain good signals by in situ hybridization, and thus, were not able to determine if these genes were expressed in a proper relative AP pattern, RT-PCR assays did indicate the relative level of their expression in these embryos (Additional file 3, Figure S3). A signal was obtained for hoxb6b, hoxb8a, hoxa9a and hoxd12, but not for hoxc13a, in $\beta$ catMO1-injected ich embryos (Additional file 3, Figure S3 - lane 3), and for all five of these genes in bmp2bMO-injected ich embryos (Additional file 3, Figure S3 - lane 8). These results show that embryos lacking both organizer and BMP signaling have the potential to express not only anterior neurectodermal markers, but also trunk posterior markers. We also tested for expression of these genes in untreated ich embryos (Additional file 3, Figure S3 - lane 2) and found that the four most posterior markers were robustly expressed. This result is consistent with the finding that ich mutant embryos do express the earlier posterior neurectodermal markers sox3 and zic2.2, which are involved in tail neural tube formation [47].

\section{AP patterning in the absence of the organizer is} modulated by the same factors as in wild-type embryos Wnt, Nodal, and FGF signaling pathways are all known to be involved in the posteriorization of neural tissue [45,54-56], an effect they achieve by epistatic interactions between themselves and the retinoic acid (RA) pathway $[45,57]$. As noted above, the absence of canonical Wnt/ $\beta$-catenin activity in ich embryos injected with bmp2bMO expands cyp26 and otx1-expressing anterior neurectoderm towards the vegetal pole in comparison with embryos treated with bmp2bMO alone (Figure 3G, J,M; Additional file 1, Figure S1G,J,M). The posterior expansion can also be seen in the comparison of $r \times 3$ expression in ich embryos injected with the three MOs and embryos injected solely with bmp2bMO (Figure 4G, $\mathrm{M})$. Also consistent with a posteriorizing role for Wnt signaling is the finding that the more posterior neurectoderm, as indicated by regions of hoxb1b, gbx 1 , and $f k d 3$ expression, is shifted markedly to a more vegetal position when ich embryos are treated either with $\beta$ catMOs alone or with $\beta$ catMOs + bmp2bMO compared to embryos injected with bmp2bMO alone (Figure $3 \mathrm{H}, \mathrm{K}, \mathrm{N}$; Additional file 1, Figure S1H,K,N; Figure 4H,K,N). These results show that the posteriorizing role of Wnt/ $\beta$-catenin signaling is completely independent of BMP signaling. That there is still ample expression of the hindbrain markers hoxb1b and $g b x 1$ in ich embryos injected with both $\beta$ catMOs suggests that other factors must act independently (or perhaps upstream of Wnt8 signaling) as posteriorizing agents. Supporting this idea is that 'ciuffo' embryos still express at least four posterior hox genes (Additional file 3, Figure S3). 
TGF $\beta$ proteins also act as posteriorizing factors during normal zebrafish development. Injection into wild-type embryos of mRNA for antivin, a potent antagonist of Nodals and Activins, results in dramatic anteriorization, with the most severely affected embryos losing all parts of the neural tube except telencephalic tissue [54]. To determine if ligands inhibited by antivin are posteriorizing factors in the absence of BMP signaling and organizer, we co-injected antivin mRNA and bmp2bMO into ich embryos. These embryos exhibited complete anteriorization of the neuroectoderm, as indicted by ubiquitous expression of the anterior markers cyp 26 and otx 1 (compare Figure 3G with 3S; Additional file 1, Figure S1G with S1S) and complete loss of the more posterior markers otx 1 and $g b x 1$ (compare Figure 3H with 3T; Additional file 1, Figure $\mathrm{S} 1 \mathrm{H}$ with $\mathrm{S} 1 \mathrm{~T}$ ). The lack of expression of the anterior markers at the vegetal pole itself is not due to an absence of expression in vegetal neurectoderm; rather, it is a consequence of a failure to complete gastrulation by those embryos co-injected with both antivin mRNA and bmp2bMO (arrowheads in Figure $3 \mathrm{~S}-\mathrm{U}$ and Additional file 1, Figure S1S-U indicate the vegetal extent of the germ-ring at $10 \mathrm{hpf}$ ), an observation consistent with a role of activin in gastrulation movements shown in Xenopus [58,59]. We also used an alternative method of inhibiting TGF $\beta$ signaling, the application of SB431542, a compound that inhibits Smad2/3-mediated TGF $\beta$-signaling [60]. Embryos grown in SB431542 also exhibited a concentration-dependent anteriorization, and also stalled during gastrulation (Additional file 1, Figure S4). Interestingly, the posteriorizing effects appear not to be entirely due to Nodal signals, as inhibiting expression of these ligands by coinjection of MOs against squint (sqt) and cyclops (cyc), the two zebrafish Nodal homologues known to be expressed during these gastrulation stages [61-64], showed much less posterior expansion of cyp26 expression compared to antivin treatment (compare Figures $2 \mathrm{G}, \mathrm{S}$ to Additional file 4, Figure S4M) and dramatically less reduction of hoxb1b expression (compare Figure $3 \mathrm{H}, \mathrm{T}$ and Additional file 4, Figure S4N).

FGF signaling is yet a third signal transduction pathway known to posteriorize neurectoderm in wild-type embryos $[16,45,65,66]$. To check if this signaling pathway also functions in embryos devoid of organizer and BMP signaling, we treated ich embryos inhibited in BMP signaling with SU5402, a small molecule inhibitor of FGF receptor activity [67]. Treatment with this compound caused the expansion of cyp 26 and $o t x 1$ anterior marker domains (compare Figure 3G with 3Y; Additional file 1, Figure S1G with S1Y) and elimination of expression of hoxblb and $g b x 1$ posterior markers (compare Figure $3 \mathrm{H}$ with 3Z; Additional file 1, Figure $\mathrm{S} 1 \mathrm{H}$ with S1Z). However, as SU5402 treatment itself appears to reduce the intensity of $c y p 26$ and hoxb $1 b$ expression, we also employed other methods of inhibiting FGF signaling (the reduction in staining intensity might be due to a role of early FGF signaling in neural capacitation [T. Kudoh, personal communication]). Injections of mRNAs encoding a dominant negative FGF receptor (XFD) [68] or the MAPK-pathway antagonist mkp3 [69] yielded a similar expansion of the cyp26 domain (Additional file 5, Figure S5M,S compared with Figure 4G), but was less effective in eliminating the hoxb1b domain (Additional file 5, Figure S5N,T compared with Figure $4 \mathrm{H})$. These latter results, however, are complicated by obvious effects on gastrulation movements that result in asymmetry of staining around the circumference of the embryo. All three FGF signaling inhibitors do, however, have dramatic anteriorizing effects on expression of neurectodermal markers.

In summary these observations show not only that embryos that fail to exhibit an early dorsal organizer show correct relative AP patterning in the absence of BMP signaling, but that this patterning is under posteriorizing control of Wnt-, TGF $\beta$-, and FGF-signaling, just as is the case in wild-type embryos.

\section{Diffuse neurectoderm surrounds mesoderm and endoderm in embryos lacking canonical Wnt signaling and impaired in BMP signaling}

As we have shown above, 24 hpf 'ciuffo' embryos are characterized by a protrusion of tissue, extending away from the yolk, that expresses a set of neurectodermal genes in proper AP order. The extension arises from abnormal and excessive epiboly movements, which causes tissue to extend far beyond the normal limit of migration, the vegetal pole of the yolk. At $24 \mathrm{hpf}$, as a consequence of this morphogenetic movement, a protruding tissue can be observed at the posterior edge of the yolk at the site of the earlier blastopore closure. As both neuroectodermal and mesodermal markers are expressed in this protrusion with overlapping patterns [8], we tested whether cells derived from each germ layer still segregate together, or not. Sections through the 'ciuffo' protrusions revealed that neuroectodermal markers (isl1, krox20) are expressed in the outer layers (Figure 5A,B), the mesodermal marker myoD in the medial layers (Figure 5B), and the endodermal marker gata5 is expressed in the innermost layer (Figure 5C). Thus, the segregation of germ layers is preserved in the protrusion of 'ciuffo' embryos. Expression of isl1 in the outer layer (Figure 5A) shows that this layer contains cells with neuronal identity, but that rather than being organized within a neural tube, they are located diffusely in a sheath of tissue that surrounds mesodermal and endodermal derivatives. Thus, when canonical Wnt signaling is absent and BMP signaling is highly reduced, 

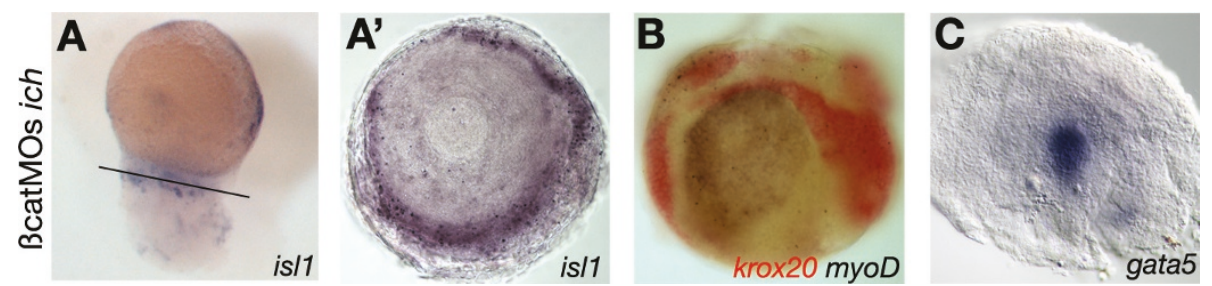

Figure 5 Germ layer segregation in 'ciuffo' embryos. Sections through the posterior protrusions of 24hpf 'ciuffo' embryos stained with characteristic germ layer markers show that neuroectoderm specific markers. islet1 (A) and krox20 (B) are expressed in the outermost layer of the protrusion. The endodermal marker gata5 (C) is expressed in the innermost layers, whereas the mesodermal marker myoD (B) can be observed in between. Sections were made of $24 \mathrm{hpf}$ ich embryos that had been injected with $\beta$ catMO1 and $\beta$ catMO2, hybridized as whole mounts with the indicated probes.

the neurectoderm retains its AP pattern and can form cells with neuronal identity, but the overall organization of this tissue is quite different from the vertebrate embryo neural tube.

\section{Discussion}

\section{Correct anteroposterior patterning in the absence of the organizer and BMP signaling}

The work described here adds to extensive evidence suggesting that an early dorsal organizer is not required for proper AP patterning of the vertebrate embryo. Surgical removal of the organizer in mouse, Xenopus, chick, and fish $[9,11-13,70]$, transplantation experiments in zebrafish [16,71], and genetic "ablations" of organizer tissue in mouse [10,14] and zebrafish [15,17,72] all strongly suggested that the organizer is not required for generation of AP pattern.

However, these earlier experiments were not definitive. The ablation experiments in chick and mice were often followed by an almost complete restoration of the organizer [11], or did not completely eliminate the expression of all organizer-related genes [13]. In the zebrafish embryo, most genes with organizer activity are expressed before the shield becomes visible; thus, physical removal of this structure can not eliminate organizer activity completely [12]. Zebrafish embryos impaired in the Nodal signaling pathway display defective shield formation [15,17], but dorsal expression of several organizer markers (e.g. chd, $g s c, b o z)$ can be observed during the development of mutant embryos [17,73], and axis formation is not completely abolished even in sqt;cyc;boz triple mutants [72].

In the zebrafish embryo, a true genetic ablation of the dorsal organizer resulting in failure to induce any dorsal markers is really only achieved by the severe reduction of maternal $\beta$-catenin- 2 in mutant ich embryos, or in embryos treated with MOs against $\beta$-catenin-2 $[7,8]$. However, as ich embryos fail to form head, trunk, and most neurectodermal tissues, their phenotype is not informative for determining if loss of dorsal organizer results in altered A-P pattern.
The lack of importance of the organizer in generating neurectodermal A-P pattern dramatically revealed only when BMP signaling is inhibited in embryos lacking the organizer. The first indication of this was the outcome of an experiment of Ober and Schulte-Merker [18] in which vegetal yolk was removed from wild-type zebrafish embryos just after fertilization. Such embryos were completely ventralized with lack of organizer (embryonic shield) and neurectoderm formation, indicating that determinants of organizer formation are localized at or near the vegetal pole. However, when this vegetal yolk removal was performed on $s w r / b m p 2 b$ embryos (which lack BMP signaling) neural tissue did form and at least two neural markers appeared with appropriate AP pattern, but were expressed radially. A similar result was obtained by comparing double boz;chd embryos, unable to form an organizer and neurectoderm, with triple boz; chd;swr embryos, in which the ectoderm is neuralized and markers of the midbrain hindbrain boundary and rhombomeres are correctly patterned [74]. In the experimental work we have presented above, we can explain the restoration of correct A-P neurectodermal pattern found in embryos inhibited in expression of both $\beta$-catenins [8] as a consequence of the inhibition of BMP signaling due to the massive ectopic expression of chd in these embryos [23]. Our results show that this patterning is equivalent to embryos lacking maternal $\beta$-catenin2 that have been inhibited in BMP signaling by injection with $b m p 2 b \mathrm{MO}$.

We also provide the first report that simultaneous inhibition of BMP signaling and organizer formation results in proper neurectodermal patterning even during gastrula stages. In Xenopus, neurectodermal markers were expressed radially in proper relative AP order in the brain of embryos lacking both organizer and BMP signaling was shown at later neurula stages, but not during gastrulation [19]. In this case, the knockdown of BMP signaling was obtained by administration of MOs against three BMPs and organizer functions were eliminated by UV treatment or administration of a $\beta$-catenin 
MO. Thus, the consequence of simultaneous elimination of both BMP signaling and organizer formation in both zebrafish and Xenopus is robust formation of radially organized neurectoderm, including all regions of the brain, with proper relative AP pattern.

It is interesting that the pattern of embryonic neurectodermal AP domains is highly conserved between chordates and the radially organized hemichordates [75,76], suggesting that the deuterostome ancestor had the same AP pattern and that generation of the AP axis is independent of the organizer. Moreover, the independence of the AP and DV axis, previously recognized in hemichordates [77], is also clearly shown in the zebrafish embryo when dorsal organizer formation is eliminated along with a reduction in BMP signaling.

\section{Multiple organizer-independent signaling pathways posteriorize the neurectoderm}

As presented in the Results section, Wnt, Nodal, and FGF signal transduction pathways, operating in the zebrafish germ-ring, posteriorize the neurectoderm in an organizer-independent manner. These findings are completely supportive of the "two-step model" of Nieuwkoop [78], with the "activating," anti-BMP signals originating from the organizer, and the "transformative" posteriorizing signals emanating from the germ-ring (Figure 6A).

It is well established that one of the major posteriorizing signals during vertebrate embryogenesis is the canonical Wnt pathway, which acts as a morphogen along the AP axis of the neural plate to regulate the expression of patterning genes [55]. Zebrafish mutants which upregulate the pathway, such as masterblind/axin1 $(m b l)$ [79] or headless/tcf3 $(h d l)$ [80] exhibit a loss of anterior neural structures, whereas impairment of Wnt signaling results in the expansion of anterior neural compartments $[45,56]$. Our results provide evidence that this posteriorizing effect of Wnt signaling is independent of the dorsal organizer. Injection of bmp2bMO alone into ich embryos clearly does not result in formation of organizer tissue (Figure 2C,F). Yet, inhibition of all detectable Wnt-signaling by the additional co-injection of the two $\beta$-catMOs [23] results in a marked expansion of anterior neuroectoderm (Figure 2M,O, Figure 3M,O). It should be noted as well that the persistence of considerable amounts of posterior markers under these conditions (Figure $2 \mathrm{~N}, 3 \mathrm{~N}$ ) suggests that Wnts are not the only posteriorizing signals and that other, parallel-acting pathways function as well.

FGFs and activin-type TGF $\beta$ signals have also been implicated in AP patterning [54,65]. Accordingly, injection of inhibitors of FGF and Nodal/Activin signaling into zebrafish embryos results in dramatic anteriorization of neural tissue $[16,45,54]$. By blocking these pathways in bmp2bMO-injected ich embryos, we were also able to induce dramatic reduction in posterior neuroectoderm and expansion of anterior neuroectodermal markers (Figure 3S-U,3Y-A', Additional file 1, Figure S1S-U,Y-A').

An alternative explanation for the observed shift in the position of the posterior markers in embryos treated with $\beta$-catMOs might be an altered posterior movement of mesodermal cells. By regulating cell-cell adhesion, the BMP gradient across the DV axis controls convergence and extension movements of lateral mesodermal cells in wild type embryos [81]. In 'ciuffo' embryos, we have demonstrated that an ectopic gradient forms between cells with active BMP signaling at the animal pole and the chd expressing cells of the germ-ring (Figure 1). This gradient is likely responsible for the excessive migration of cells towards the vegetal pole and the formation of the characteristic protrusions at $24 \mathrm{hpf}$. However, as triple morphant (bmp2bMO and the two $\beta$ catMOs) embryos show an almost identical posteriorization as 'ciuffo' embryos, without any sign of abnormal posterior cell movements, we think it unlikely that the posteriorization in 'ciuffo' embryos is due to such movements. We followed the post-gastrulation development of untreated and morphant ich embryos and although excess tissue can be readily observed at the vegetal pole of 'ciuffo' embryos at 12.5-13.5 hpf, the triple morphants resemble ich embryos treated with bmp2bMO alone, with tissue accumulation at both animal and vegetal sides (Additional file 6, Figure S6).

The "two step model" provides a comprehensive explanation for our observations (Figure 6). When the "activating", BMP-antagonist signals are absent, the effects of the "transformative" signals, although present, are masked by the lack of the neuroectoderm. This is the case in ich embryos, which lack expression of BMP antagonists and in which presence of BMPs is ubiquitous (Figure 6B). In contrast, the removal of BMP-signaling transforms the complete ectoderm to neuroectodermal fate, and patterning domains in ich embryos appear as circumferential rings (Figure 6E). The normal "transformative" posteriorizing signals clearly operate in these embryos but are not restricted by the ventral cues that would have functioned in the wild-type state. Under such conditions an impairment in the level of posteriorizing factors can be easily observed as a vegetal "shift" (anteriorization) in the position of neuroectodermal expression domains in ich embryos. The shift can be caused by inhibition of germ-ring Wnt8 signaling by treatment with both $\beta$-catMOs (Figure 6F), or by inhibition of Nodal/Activin with antivin or SB431542, or by inhibition of FGF signaling with SU5402 or mkp3 or XFD (Figure 6G). However, in the case of ich embryos treated with these Nodal/Activin or 

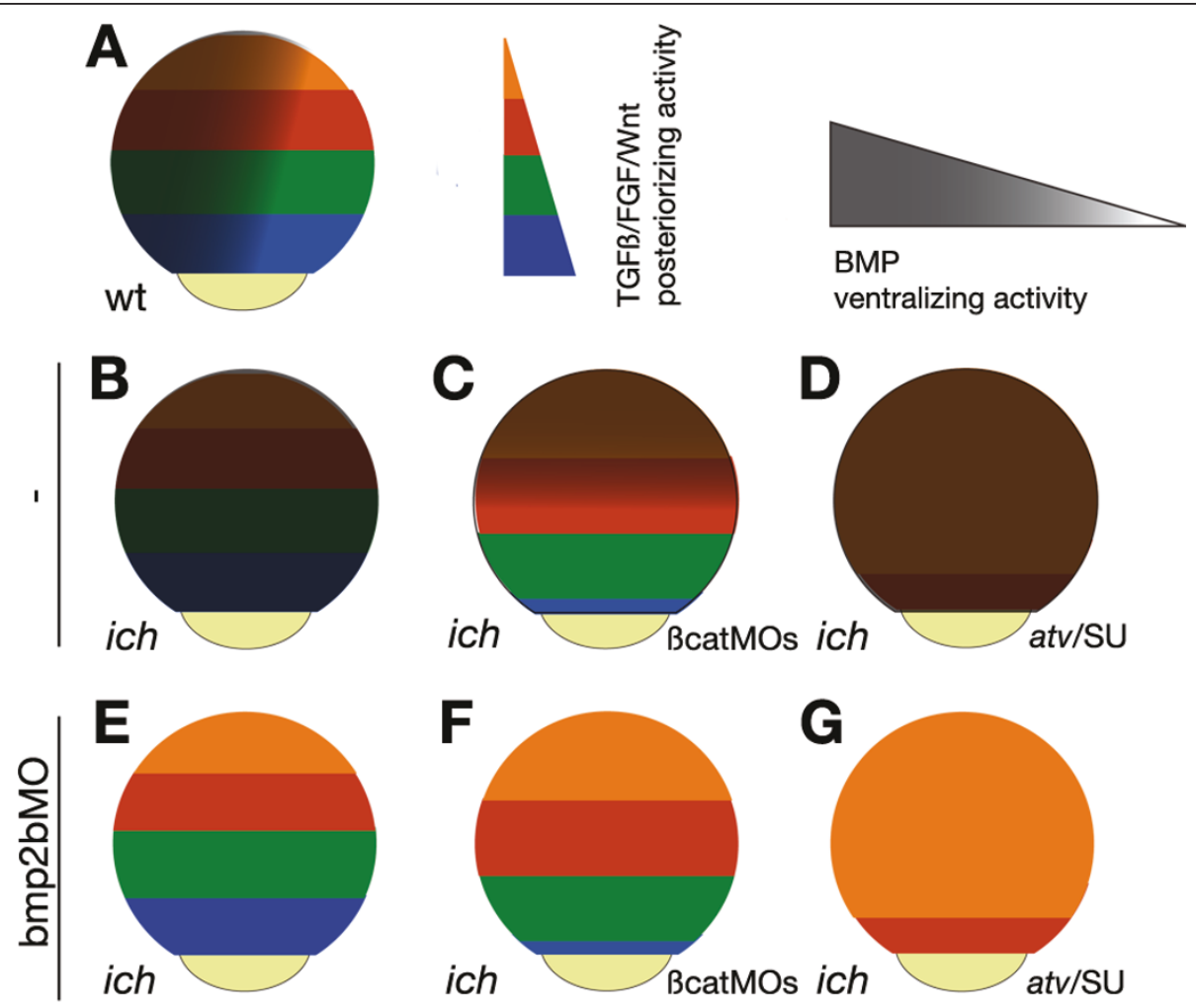

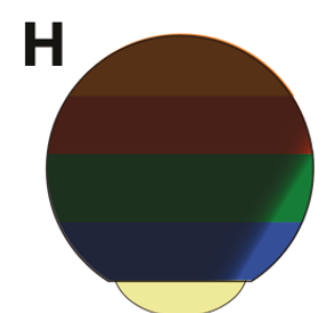

ich (C2)

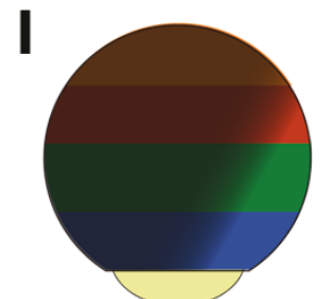

ich (C3)

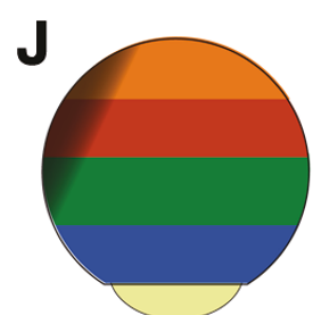

swr/sbn/snh

Figure 6 Patterning of the zebrafish neurectoderm is achieved by the parallel action of posteriorizing "transformative" signals and "activating" BMP-antagonists. (A-J) Neurectodermal regions are color coded as indicated with the most anterior territory represented in orange and the most posterior in blue. The BMP signaling gradient is indicated by grey shading, so that a brownish shading superimposed on the colored regions represent areas of the embryo unable to form neurectoderm due to the absence of an "activating" signal. Embryos of indicated genotype and treatment are diagrammed at 80-90\% epiboly. See text for details.

FGF inhibitors, the anteriorization is only observed in embryos also inhibited in BMP signaling (Figure 6G) as, otherwise, the ubiquitous expression of BMPs prevents formation of any neurectoderm at all (Figure 6D). 'Ciuffo' (i.e., $\beta$ cat1MO-treated ich) embryos (Figure 6C) present a state quite similar to bmp2bMO-treated ich embyros, as the lack of canonical Wnt signaling induces large amounts of BMP-antagonists in a wide band extending from the germ-ring, allowing the "transformative" Nodal/Activin and FGF signals to operate. However, such embryos still retain BMP signaling in the animal pole region of the embryos and thus, neurectodermal markers are not expressed in the animal-most fourth of the embryo.
This model (which is similar to the one proposed earlier by Meinhardt based on theoretical considerations [5], and more recently by Niehrs based on the review of the existing literature [82]) also offers an explanation for multiple aspects of the dorsalized and ventralized phenotypes described in several zebrafish mutants. For example, the progressive loss of anterior neural tissue observed in embryos with different degrees of ventralization, such as less severe ich embryos $(\mathrm{C} 2, \mathrm{C} 3, \mathrm{C} 4)$ [7] or chd and nog1 double deficient embryos (CI,CII) [83], is due to the smaller size of the "activation" domain and not to a change in the "transformative" signal (Figure $6 \mathrm{H}, \mathrm{I})$. As anti-BMP signals fail to reach the animal-most region of the gastrula, markers of the forebrain (and 
often midbrain) will not be turned on. Similarly, in mutants of the BMP pathway, such as swr, somitabun $(s b n)$ and snailhouse (snh) [30], the oversized BMPabsent area results in laterally expanded, but otherwise correctly positioned, expression domains during gastrulation (Figure 4J) $[32,84]$, indicating that the "transformative" signal is not perturbed in such embryos.

Our observations clearly refute the argument that the zebrafish AP and "classical" DV axes are equivalent $[20,21]$, as it is clear from work presented here that the prospective AP axis of the embryo coincides with the early animal-vegetal axis of the zygote. As Xenopus embryos deficient both in organizer formation and BMP signaling also express AP patterning markers circumferentially [19], we propose that the concordance of the AP and animal-vegetal axis is a general feature of the anamniote embryos.

\section{'Ciuffo' embryos may reveal ancestral anteroposterior radial neurectoderm patterning}

Two striking features of the neurectoderm of $24 \mathrm{hr}$ 'ciuffo' embryos are the radial organization along the full AP extent of the embryo, and the location of cells of neuronal identity in a diffuse network close to the outer surface, while tissues of mesodermal and endodermal identity reside within the embryo. This organization is reminiscent of the supposed pre-Urbilaterian, protoEumetazoan ancestor. Comparative studies suggest that the Eumetazoan ancestor had a diffuse nerve net [85] and members of Cnidaria, the sister clade of Eumetazoa, still possess such a primitive nervous system. Although it is yet unclear how exactly the patterning of this structure occurs, it has been suggested that FGF and TGF $\beta$ signaling pathways play an active role in specifying the neuronal identities observed in Nematostella vectensis, an anthozoan cnidarian [86]. This view coincides with the evolutionary scenario suggested by Arendt et al. [87] in which BMP signaling was originally involved in the patterning of neuronal cell types and it was only adapted later to control formation of a centralized nervous system. Indeed, in several anthozoan species, asymmetric expression of BMPs and BMP antagonists has been observed, suggesting both that the origins of the bilaterian DV patterning system predate the Cnidarian Eumetazoan split and that the system was originally not involved in driving nervous system centralization [88-91]. Strikingly, in Nematostella most BMP-components are expressed in the endoderm [90] and recent functional data indicates a major role in endoderm patterning (and a lesser role in neural differentiation) for the BMP pathway [92]. It is noteworthy that chordin and different $b m p / d p p$ genes are expressed on the same side of the directive axis in Nematostella, suggesting a patterning center with functional similarities to the chd/ admp-expressing vertebrate organizer [92]. Such a center alone is sufficient to create a BMP gradient in the embryo [93], most likely through the shuttling of Chd/ BMP complexes [94]. As a similar system was recently described for sea urchins as well [95], we suggest that this might constitute a prototypical BMP signaling paradigm, which later evolved into the scalable BMP signaling system observed in some Bilaterians.

Interestingly, some larval hemichordates (which together with echinoderms form the sister group of vertebrates in Deuterostoma) also possess a diffuse, epidermal neural network (reviewed in Gerhart et al. [96]). New research, however, suggests that this feature is not homologous with the vertebrate neural plate (as has been previously suggested [77]), but is a transient larval adaptation of certain hemichordate species [97]. As whenever a CNS is present in Bilateria, it develops on the side of BMP antagonism [87], it can be inferred that a centralized nervous system, regulated by BMP signals, is the ancestral state for all bilaterians. In echinoderms and certain hemichordates, where centralization is not observed, a secondary loss might have occurred during the evolution of these lineages [98]. By eliminating Wnt signaling and greatly reducing BMP signaling in 'ciuffo' embryos, we can observe the lack of restriction of neurectoderm to a particular DV level. That the potential for such a "diffuse nerve net" exists in a chordate species suggests that the evolutionary transition between a centralized and diffuse nervous system might have involved only a quite limited number of steps.

The organizer apparently arose in a chordate ancestor, as a signaling center homologous to the vertebrate organizer has been found in the cephalochordate (amphioxus) Branchiostoma floridae [99]. As tunicates are now considered the sister group of vertebrates, and amphioxus is more distantly related, it is likely that the organizer was lost in tunicates [99]. Chordate embryos have evolved the organizer to provide among other signaling functions, a specialized temporal and spatial program of BMP antagonist expression which acts on a pre-existing, extremely highly conserved pattern of AP tissue specification. As the AP pattern of gene expression appears to be widely conserved throughout the animal kingdom, it will be of interest to test whether the same pattern-generating "transformative" signals operate in hemichordates and non-vertebrate chordates. Wnt signals have already been shown in cephalochordates (amphioxus) to be expressed posteriorly, around the germ-ring, very much as in fish and amphibians, indicating that Wnts are a posterior "transformative" signal characteristic of chordates [99-101]. In amphioxus, the effects of this signal appear to be strongest at the posterior end of the embryo, while retinoic acid has a more important role in determining AP identity elsewhere 
[100]. Wnt signaling is an extremely ancient mechanism of patterning the body axis [82,102], as it operates to specify position along the main body axis of the cnidarian Nematostella vectensis (a sea anemone) [103,104]. There is virtually nothing known, however, about FGF and Nodal signaling as potential "transformative" signals in non-vertebrate embryos.

\section{Conclusions}

Our work provides evidence for the organizer-independent AP patterning of the neuroectoderm in the developing zebrafish gastrula. We observed correctly located AP neurectodermal domains in the organizer-less ich mutant embryos when BMP-signaling was inhibited. The position and size of these domains depends mainly on the action of Wnt-, FGF- and Nodal signaling, originating from the germ-ring of the gastrula. These observations can be easily interpreted within the framework of Nieuwkoop's "two step model": the observed neurectodermal pattern in wild type fish is the result of the concerted action of "activating" and "transformative" signals (in the case of the zebrafish, BMP-antagonists and germ-ring-derived morphogens, respectively).

Our results also clearly refute recent proposals about the equivalency of the AP and "classical" DV axes in anamniotes, as the concordance of the AP axis with the AnVeg axis of the early embryo is evident.

When both Wnt- and BMP-signaling was inhibited in ich embryos, they developed into a well patterned tubelike structure, where a neurectodermal sheet envelopes inner mesodermal and endodermal tissues. In this neurectodermal domain scattered neuronal progenitors can be detected. Therefore, we speculate that vertebrates still retain the genetic program to form an ancient radially-organized diffuse neural net, and that only a limited number of changes in this program may have been necessary to form a neural-tube type of organization.

\section{Methods}

\section{Zebrafish strains and husbandry}

Zebrafish were maintained under standard conditions [105]. Wild-type embryos were derived from AB parents, while ichabod ${ }^{1}$ (ich) embryos were obtained by breeding homozygous ich females with brass or ich males. Only those ich females that reproducibly yielded severely ventralized Class 1 (or Class 1a) phenotypes (for details see $[7,40]$ ) were used. All animal work described here was carried out under Protocols 700433 and 801973 approved by the Institutional Animal Care and Use Committee (IACUC) of the University of Pennsylvania. This committee approves animal work only if it follows internationally recognized ethical and experimental guidelines.

\section{Morpholino antisense oligonucleotide and mRNA injections}

$\beta$-catenin-1-MO ( $\beta$ cat1MO) [8], $\beta$-catenin-2-MO ( $\beta$ cat $2 \mathrm{MO})$ [8], bmp2bMO [26], sqtMO [106], and cycMO [107] were manufactured by Gene Tools (Philomath, OR) and the sequences of each are as published. Clones used to prepare sense mRNA were as follows: mkp3 in pCS2+ [69] and antivin (lefty1) in pCS2+ [108]. mRNAs were synthesized and capped using an mMessage mMachine Kit (Ambion), following the manufacturer's protocol. RNAs and morpholinos were stored in $\mathrm{dH}_{2} \mathrm{O}$ at $-20^{\circ} \mathrm{C}$ and injection solutions were prepared by diluting a $2 \times$ stock of reagent in $\mathrm{dH}_{2} \mathrm{O}$ with an equal volume of Dulbecco's modified phosphate-buffered saline containing 5\% phenol red (Sigma). Approximately 1 $\mathrm{nl}$ of solution was injected into the yolk at the base of blastomeres of 1-4 cell embryos. The concentration of these antisense and sense reagents that were injected per embryo were as follows: $\beta$ cat $1 \mathrm{MO}$ and $\beta$ cat $2 \mathrm{MO}(\beta$ catMOs) (3 mM each), bmp2bMO (0.4 mM), sqtMO and cycMO (0.6 mM each), and antivin (atv) mRNA (200 ng/ $\mu \mathrm{l})$.

\section{Small molecule treatments}

SB431542 (Sigma) and SU5402 (Calbiochem) were used at indicated concentrations to block TGF $\beta$-activin and FGF signaling, respectively. The reagents were dissolved in DMSO and added to the culture medium at the 1000-cell stage. Embryos were kept in the dark until fixed.

\section{RT-PCR experiments}

Total RNA isolated from pooled samples of 10 embryos was used to generate cDNA using the SuperScript II kit (Invitrogen), following the manufacturer's protocol. The primers used in the experiment were as follows (all shown in 5'-3' orientation): krox20-F: CTGCCAGC CTCTGTGACTA, krox20-R: CCATGGTGCAGCTGA GAGT, hoxb6b-F: CTGACCGCTCGTGCGCTAT, hoxb6b-R: ATCTTCCTCATCGCTGACCTT, hoxb8a-F: GCAGAGTCCATGTGCGGTAA, hoxb8a-R: CAATCC GACGCTTGCGTGTT, hoxa9a-F: AACTGAGCCACCGACGGTTA, hoxa9a-R: TCTTCATCCTGCGGTTTT GGA, hoxd12-F: GTCACTGAGCGCCCAGAAT, hoxd12-R: CAGTTCCAATCTGTCCGAAA, hoxc13a-F: CCACGTCACGATGCATTGAT, hoxc13a-R: TAACTTGACGTTCTGAGAGGTT, ef1a-F: ACCGGCCATCTGATCTACAA and ef1a-R: CAATGGTGATACCACG CTCA.

\section{Whole mount in situ hybridizations and immunohistochemistry}

The following clones were used to prepare antisense probes for hybridization: p63 [35], krox20 [53], emx1 
[109], val (D. Grunwald, cDNA library), cyp26 [45], hoxb1b [46], hoxb6b [110], otx1 [48], $g b x 1$ [49], $r x 3$ [50], boz [38], gsc [25], chd [111] and fkd3 [51]. Antisense RNA probes were synthesized and two color in situ hybridization was carried out as previously described [23]. For embryos older than 24 hpf (Figure 5; Additional file 1, Figure S1) NBT/BCIP was used as primary chromogen, as it gave better results. Whole mount embryos were imaged with a Leica MZ12 stereomicroscope, using a Roper Scientific Photometrics RGB Vision MS-C digital camera system (CRI, Inc, Boston, MA).

For manual sections (Figure 5) embryos subjected to whole-mount in situ hybridisation were cleared in serial incubations of glycerol (25, 50, 75 and 95\%). Sections were placed in a drop of glycerol, cover-slipped, and imaged with $40 \times(0.8 \mathrm{NA})$ water-immersion lens using a Nikon E1000 microscope connected to a digital camera (Jenoptik) operated by Openlab (Improvision) software.

For immunohistochemistry, embryos were fixed in $4 \%$ PFA, blocked in standardized blocking solution (10\% fetal bovine serum, $1 \%$ DMSO, $0.8 \%$ Triton- $\mathrm{X}$ in PBS), incubated overnight at $4 \mathrm{C}^{\circ}$ with a 1:100 dilution of anti-phospho-Smad1/5/8 antibody (Cell Signaling Technology), followed by a 1:200 dilution of goat anti-rabbit Alexa Fluor 488-conjugated antibody (Invitrogen). To visualize the nuclei, embryos were incubated in 1:1000 dilution of TO-PRO ${ }^{\circledR}-3$ iodide (Invitrogen) DNA stain in PBST. After several washes the specimens were mounted in $1 \%$ low melting point agarose, and imaged on a Leica TCS SP Confocal Microscope using $\times 10$ objectives. Image reconstruction was performed using Volocity (Improvison) software.

All figures were composed using Adobe Photoshop and Illustrator (CS3).

\section{Additional material}

Additional file 1: Figure S1 - Wnt-, TGF $\beta$ - and FGF-signaling have pivotal roles in the posteriorization of the neuroectoderm of embryos both inhibited in BMP signaling and devoid of organizer activity. Single in situ hybridization with ot $x 1(A, D, G, J, M, P, S, V, Y)$ or $g b \times 1$ $(B, E, H, K, Q, T, W, Z)$ probes or double in situ hybridization with both probes $\left(C, F, I, L, O, R, U, X, A^{\prime}\right)$ is shown for wild-type embryos (A-C), untreated ich embryos (D-F), or ich embryos treated with bmp2bMO (G-I), the two $\beta$ cat MOs (J-L), bmp2bMO plus the two $\beta$-cat MOs (M-O), or ich embryos injected with antivin mRNA (P-R), antivin mRNA plus bmp2bMO (S-U), SU5402 (V-X), or SU5402 plus bmp2bMO (Y-A'). Wild-type embryos [A-C] are shown in dorsal views, while ich embryos are shown in lateral views. All embryos are at $~ 100 \%$ epiboly. Arrowheads in the antivin (atv) treated embryos point to the edge of the germ-ring.

Additional file 2: Figure S2 - Complete repression of $\beta$-catenin signaling in ich embryos induces neuroectoderm with correct AP pattern. Untreated ich embryos do not express emx1, krox20, val, or hoxb6b $(B, E, H)$, while their siblings coinjected with $\beta$ catMOs $\left(C, C^{\prime}, F, F^{\prime}, I_{1}\right)$ express these neuroectodermal markers in a correct order at $22 \mathrm{hpf}$, with the anterior to posterior direction corresponding to proximal to distal relative to the yolk (compare A with $C, C^{\prime}, D$ with $F$, F', and $G$ with $I^{\prime} \prime^{\prime}$ ).
The following probe-pairs were used: $e m \times 1$ (blue) and krox20 (red) (A-C'), krox20 (red) and val (blue) (D-F'), and krox20 (red) and hoxb6b (blue) (G-l'),

Additional file 3: Figure S3 - Inhibition of Wnt- or BMP signaling in ich embryos induces typical anterior neurectodermal markers. RTPCR amplification of characteristic neurectodermal patterning markers (krox20, hoxb6b, hoxb8a, hoxa9a, hoxd12, hoxc13a) was carried out using oligo dT-primed CDNA samples from wild-type embryos (lanes 1-4), uninjected ich embryos (lanes 2,5,7,9), or ich embryos injected with $\beta$ cat1MO ('ciuffo') (lanes 3,6 ) or bmp2bMO embryos (lanes 8,10 ). Whereas in ich embryos, only the posterior-most hox markers are present (lanes $2,7)$, 'ciuffo' embryos expressed almost the complete range of neurectodermal patterning markers examined, except that expression of hoxc13a, the most posterior marker examined, is absent, and the level of hoxd12a is reduced (lane 3). In contrast, bmp2bMO injection induced expression of all assayed neurectodermal markers (lane 8). Controls lacking RT were performed to make sure the signals observed were dependent on RNA (lanes 4-6,9,10).

Additional file 4: Figure S4 - Posteriorizing TGF $\beta$ activity is only partly dependent on Nodal signals. In situ hybridization with cyp26 (A, $C, E, G, I, K, M)$ or hoxb1b $(B, D, F, H, J, L, N)$ probes is shown for wild-type embryos $(A, B)$, untreated ich embryos $(C, D)$, ich embryos treated with bmp2bMO (E,F), SB431542 $(\mathrm{G}, \mathrm{H})$, sqtMO and cycMO $(\mathrm{K}, \mathrm{L})$, or bmp2bMO in combination with SB431542 (I,J) or sqtMO and cyc $M O(M, N)$. A small molecular inhibitor of the TGF $\beta$ pathway, SB431542, has no effect on untreated ich embryos $(\mathrm{G}, \mathrm{H})$, but when it is applied to bmp2bMO injected ich embryos, it anteriorizes the neuroectoderm $(I, J)$. These embryos have gastrulation defects; the arrowheads point to the position of the stalled germ-ring. Coinjection of sqtMO and cycMO with bmp2bMO results in a slight expansion of the cyp26 domain (M), and a mild reduction of the hoxblb domain $(\mathrm{N})$, showing that the posteriorizing effects of TGF $\beta$ signaling are dependent to some extent on Nodal-independent signals. Injection of sqtMO along with cycMO into untreated ich embryos has no effect on cyp26 and hoxb1b expression (K, L). SB431542 was used at $2.4 \mathrm{mM}$ concentration; sqtMO and cycMO were $3 \mathrm{mM}$ each. Wild-type embryos $(A, B)$ are shown in a dorsal view, ich embryos (C-N) from a lateral view. All embryos are at $\sim 10 \mathrm{hpf}$.

Additional file 5: Figure S5 - Antagonists of the FGF pathway can anteriorize ich embryos with impaired BMP signaling. Single in situ hybridization with cyp26 $(\mathrm{A}, \mathrm{D}, \mathrm{G}, \mathrm{J}, \mathrm{M}, \mathrm{P}, \mathrm{S})$ or hoxb1b $(\mathrm{B}, \mathrm{E}, \mathrm{H}, \mathrm{K}, \mathrm{N}, \mathrm{Q}, \mathrm{T})$ probes or double in situ hybridization with both probes $(C, F, I, L, O, R, U)$ is shown for wild-type embryos (A-C), untreated ich embryos (D-F), ich embryos treated with bmp2bMO (G-I), injected with XFD or mkp3 mRNAs alone (J$\mathrm{L}$ and $\mathrm{P}-\mathrm{R}$ ), or in combination with bmp2bMO (M-O and S-U). The injection of mRNAs encoding a dominant negative FGF receptor, XFD, or a negative regulator of the MAPK pathway, mkp3, into bmp2bMOinjected embryos results in the posterior expansion of the anterior neurectodermal marker, cyp26 $(\mathrm{M}, \mathrm{O}$, and $\mathrm{S}, \mathrm{U}$, and a reduction of the posterior neurectodermal domain, marked by hoxbib ( $N, O$ and $T, U)$. Gastrulation movements seem to be impaired in such coinjected embryos. These antagonists of FGF signaling have no effect on untreated ich embryos ( $\mathrm{J}-\mathrm{L}$ and $\mathrm{P}-\mathrm{R})$. Wild-type embryos (A-C) are shown in a dorsal view, ich embryos (D-U) in lateral view. All embryos are at $\sim 10 \mathrm{hpf}$.

Additional file 6: Figure S6 - Posterior movement of mesodermal cells only observed in 'ciuffo' embryos. Lateral views of live, 12.5-13 hpf ich embryos untreated (A) or treated with bmp2bMO (B), Bcat1MO (C) and bmp2bMO and $\beta$ cat $1 \mathrm{MO}$ (D). Note the clear vegetal migration of cells observable in 'ciuffo' embryos (C), and the relatively symmetric distribution of tissues between the animal and vegetal poles of bmp2bMO (co-)injected embryos (B,D).

\section{Acknowledgements}

We thank Joshua Bradner for fish care and technical help, Gianfranco Bellipanni for helpful and stimulating discussions and Steve Wilson and Jon Clarke for gifts of SB431542 and SU5402. This work was supported by NIH grant R01 HD39272 to E.S.W. 


\section{Author details}

'Department of Biology, University of Pennsylvania, Philadelphia, PA 19104 USA. ${ }^{2}$ Current Address: Department of Genetics, Eötvös Loránd University, Pázmány Péter sétány $1 / C$, Budapest H-1117, Hungary. ${ }^{3}$ Current Address: Dept. of Intelligence Science and Technology, Graduate School of Informatics, Kyoto University, Yoshidia-Honmachi, Sakyo, Kyoto 606-8501, Japan.

\section{Authors' contributions}

MV conducted the experimental design and execution, and drafted the manuscript. SM performed some of the morpholino injection and in situ hybridization experiments. ESW oversaw the design of the experiments and the writing the final draft the manuscript. All authors read and approved the final manuscript.

Received: 4 January 2011 Accepted: 16 May 2011

Published: 16 May 2011

\section{References}

1. Harland R, Gerhart J: Formation and function of Spemann's organizer. Annu Rev Cell Dev Biol 1997, 13:611-67.

2. De Robertis EM, Kuroda H: Dorsal-ventral patterning and neural induction in Xenopus embryos. Annu Rev Cell Dev Biol 2004, 20:285-308.

3. Schier AF, Talbot WS: Molecular genetics of axis formation in zebrafish. Annu Rev Genet 2005, 39:561-613.

4. Stern CD, Charite J, Deschamps J, Duboule D, Durston AJ, Kmita M, Nicolas JF, Palmeirim I, Smith JC, Wolpert L: Head-tail patterning of the vertebrate embryo: one, two or many unresolved problems? Int I Dev Biol 2006, 50:3-15.

5. Meinhardt H: Primary body axes of vertebrates: generation of a nearCartesian coordinate system and the role of Spemann-type organizer. Dev Dyn 2006, 235:2907-19.

6. Heasman J, Kofron M, Wylie C: Beta-catenin signaling activity dissected in the early Xenopus embryo: a novel antisense approach. Dev Biol 2000, 222:124-34.

7. Kelly C, Chin AJ, Leatherman JL, Kozlowski DJ, Weinberg ES: Maternally controlled (beta)-catenin-mediated signaling is required for organizer formation in the zebrafish. Development 2000, 127:3899-911.

8. Bellipanni G, Varga M, Maegawa S, Imai Y, Kelly C, Myers AP, Chu F, Talbot WS, Weinberg ES: Essential and opposing roles of zebrafish betacatenins in the formation of dorsal axial structures and neurectoderm. Development 2006, 133:1299-309.

9. Snow MH: Autonomous development of parts isolated from primitivestreak-stage mouse embryos. Is development clonal? I Embryol Exp Morphol 1981, 65(Suppl):269-87.

10. Ang SL, Rossant J: HNF-3 beta is essential for node and notochord formation in mouse development. Cell 1994, 78:561-74.

11. Psychoyos D, Stern CD: Restoration of the organizer after radical ablation of Hensen's node and the anterior primitive streak in the chick embryo. Development 1996, 122:3263-73.

12. Shih J, Fraser SE: Characterizing the zebrafish organizer: microsurgical analysis at the early-shield stage. Development 1996, 122:1313-22.

13. Davidson BP, Kinder SJ, Steiner K, Schoenwolf GC, Tam PP: Impact of node ablation on the morphogenesis of the body axis and the lateral asymmetry of the mouse embryo during early organogenesis. Dev Biol 1999, 211:11-26.

14. Klingensmith J, Ang SL, Bachiller D, Rossant J: Neural induction and patterning in the mouse in the absence of the node and its derivatives. Dev Biol 1999, 216:535-49.

15. Feldman B, Gates MA, Egan ES, Dougan ST, Rennebeck G, Sirotkin HI, Schier AF, Talbot WS: Zebrafish organizer development and germ-layer formation require nodal-related signals. Nature 1998, 395:181-5.

16. Koshida S, Shinya M, Mizuno T, Kuroiwa A, Takeda H: Initial anteroposterior pattern of the zebrafish central nervous system is determined by differential competence of the epiblast. Development 1998, 125:1957-66.

17. Gritsman K, Zhang J, Cheng S, Heckscher E, Talbot WS, Schier AF: The EGFCFC protein one-eyed pinhead is essential for nodal signaling. Cell 1999, 97:121-32.

18. Ober EA, Schulte-Merker S: Signals from the yolk cell induce mesoderm, neuroectoderm, the trunk organizer, and the notochord in zebrafish. Dev Biol 1999, 215:167-81.
19. Reversade B, Kuroda H, Lee H, Mays A, De Robertis EM: Depletion of Bmp2, Bmp4, Bmp7 and Spemann organizer signals induces massive brain formation in Xenopus embryos. Development 2005, 132:3381-92.

20. Lane MC, Sheets MD: Rethinking axial patterning in amphibians. Dev Dyn 2002, 225:434-47.

21. Lane MC, Sheets MD: Heading in a new direction: implications of the revised fate map for understanding Xenopus laevis development. Dev Biol 2006, 296:12-28.

22. Gerhart J: Changing the axis changes the perspective. Dev Dyn 2002, 225:380-3.

23. Varga M, Maegawa S, Bellipanni G, Weinberg ES: Chordin expression, mediated by Nodal and FGF signaling, is restricted by redundant function of two beta-catenins in the zebrafish embryo. Mech Dev 2007, 124:775-91.

24. Piccolo S, Sasai Y, Lu B, De Robertis EM: Dorsoventral patterning in Xenopus: inhibition of ventral signals by direct binding of chordin to BMP-4. Cell 1996, 86:589-98.

25. Stachel SE, Grunwald DJ, Myers PZ: Lithium perturbation and goosecoid expression identify a dorsal specification pathway in the pregastrula zebrafish. Development 1993, 117:1261-74.

26. Imai Y, Talbot WS: Morpholino phenocopies of the bmp2b/swirl and bmp7/snailhouse mutations. Genesis 2001, 30:160-3.

27. Faure $S$, Lee MA, Keller T, ten Dijke P, Whitman M: Endogenous patterns of TGFbeta superfamily signaling during early Xenopus development. Development 2000, 127:2917-31.

28. Schohl A, Fagotto F: Beta-catenin, MAPK and Smad signaling during early Xenopus development. Development 2002, 129:37-52.

29. Tucker JA, Mintzer KA, Mullins MC: The BMP signaling gradient patterns dorsoventral tissues in a temporally progressive manner along the anteroposterior axis. Dev Cell 2008, 14:108-19.

30. Mullins MC, Hammerschmidt M, Kane DA, Odenthal J, Brand M, van Eeden FJ, Furutani-Seiki M, Granato M, Haffter P, Heisenberg CP, et al: Genes establishing dorsoventral pattern formation in the zebrafish embryo: the ventral specifying genes. Development 1996, 123:81-93.

31. Kishimoto Y, Lee KH, Zon L, Hammerschmidt M, Schulte-Merker S: The molecular nature of zebrafish swirl: BMP2 function is essential during early dorsoventral patterning. Development 1997, 124:4457-66.

32. Nguyen VH, Schmid B, Trout J, Connors SA, Ekker M, Mullins MC: Ventral and lateral regions of the zebrafish gastrula, including the neural crest progenitors, are established by a bmp2b/swirl pathway of genes. Dev Biol 1998, 199:93-110.

33. Schmid B, Furthauer M, Connors SA, Trout J, Thisse B, Thisse C, Mullins MC: Equivalent genetic roles for bmp $7 /$ snailhouse and $\mathrm{bmp} 2 \mathrm{~b} / \mathrm{swirl}$ in dorsoventral pattern formation. Development 2000, 127:957-67.

34. Munoz-Sanjuan I, Brivanlou AH: Neural induction, the default model and embryonic stem cells. Nat Rev Neurosci 2002, 3:271-80.

35. Cruz C, Maegawa S, Weinberg ES, Wilson SW, Dawid IB, Kudoh T: Induction and patterning of trunk and tail neural ectoderm by the homeobox gene eve1 in zebrafish embryos. Proc Natl Acad Sci USA 2010, 107:3564-9.

36. Yamanaka Y, Mizuno T, Sasai Y, Kishi M, Takeda H, Kim CH, Hibi M, Hirano T: A novel homeobox gene, dharma, can induce the organizer in a noncell-autonomous manner. Genes Dev 1998, 12:2345-53.

37. Fekany K, Yamanaka Y, Leung T, Sirotkin HI, Topczewski J, Gates MA, Hibi M, Renucci A, Stemple D, Radbill A, et al: The zebrafish bozozok locus encodes Dharma, a homeodomain protein essential for induction of gastrula organizer and dorsoanterior embryonic structures. Development 1999, 126:1427-38.

38. Koos DS, Ho RK: The nieuwkoid gene characterizes and mediates a Nieuwkoop-center-like activity in the zebrafish. Curr Biol 1998, 8:1199-206.

39. Reifers F, Bohli H, Walsh EC, Crossley PH, Stainier DY, Brand M: Fgf8 is mutated in zebrafish acerebellar (ace) mutants and is required for maintenance of midbrain-hindbrain boundary development and somitogenesis. Development 1998, 125:2381-95.

40. Maegawa S, Varga M, Weinberg ES: FGF signaling is required for \{beta\}catenin-mediated induction of the zebrafish organizer. Development 2006, 133:3265-76

41. Dixon Fox M, Bruce AE: Short- and long-range functions of Goosecoid in zebrafish axis formation are independent of Chordin, Noggin 1 and Follistatin-like 1b. Development 2009, 136:1675-85. 
42. Glinka A, Wu W, Onichtchouk D, Blumenstock C, Niehrs C: Head induction by simultaneous repression of Bmp and Wnt signalling in Xenopus. Nature 1997, 389:517-9.

43. Oelgeschlager M, Kuroda $H$, Reversade B, De Robertis EM: Chordin is required for the Spemann organizer transplantation phenomenon in Xenopus embryos. Dev Cell 2003, 4:219-30.

44. Imai Y, Gates MA, Melby AE, Kimelman D, Schier AF, Talbot WS: The homeobox genes vox and vent are redundant repressors of dorsal fates in zebrafish. Development 2001, 128:2407-20.

45. Kudoh T, Wilson SW, Dawid IB: Distinct roles for Fgf, Wnt and retinoic acid in posteriorizing the neural ectoderm. Development 2002, 129:4335-46

46. Alexandre D, Clarke JD, Oxtoby E, Yan YL, Jowett T, Holder N: Ectopic expression of Hoxa-1 in the zebrafish alters the fate of the mandibular arch neural crest and phenocopies a retinoic acid-induced phenotype. Development 1996, 122:735-46.

47. Kudoh T, Concha ML, Houart C, Dawid IB, Wilson SW: Combinatorial Fgf and Bmp signalling patterns the gastrula ectoderm into prospective neural and epidermal domains. Development 2004, 131:3581-92.

48. Li $Y$, Allende ML, Finkelstein R, Weinberg ES: Expression of two zebrafish orthodenticle-related genes in the embryonic brain. Mech Dev 1994 48:229-44

49. Rhinn M, Lun K, Amores A, Yan YL, Postlethwait JH, Brand M: Cloning, expression and relationship of zebrafish gbx1 and gbx2 genes to Fgf signaling. Mech Dev 2003, 120:919-36.

50. Chuang JC, Mathers PH, Raymond PA: Expression of three Rx homeobox genes in embryonic and adult zebrafish. Mech Dev 1999, 84:195-8.

51. Odenthal J, Nusslein-Volhard C: fork head domain genes in zebrafish. Dev Genes Evol 1998, 208:245-58.

52. Pfeffer PL, Gerster T, Lun K, Brand M, Busslinger M: Characterization of three novel members of the zebrafish Pax2/5/8 family: dependency of Pax5 and Pax8 expression on the Pax2.1 (noi) function. Development 1998, 125:3063-74

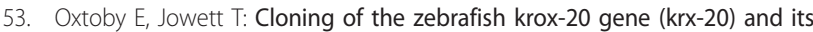
expression during hindbrain development. Nucleic Acids Res 1993, 21:1087-95.

54. Thisse B, Wright CV, Thisse C: Activin- and Nodal-related factors control antero-posterior patterning of the zebrafish embryo. Nature 2000 403:425-8.

55. Kiecker C, Niehrs C: A morphogen gradient of Wnt/beta-catenin signalling regulates anteroposterior neural patterning in Xenopus. Development 2001, 128:4189-201.

56. Erter CE, Wilm TP, Basler N, Wright CV, Solnica-Krezel L: Wnt8 is required in lateral mesendodermal precursors for neural posteriorization in vivo. Development 2001, 128:3571-83.

57. White RJ, Nie Q, Lander AD, Schilling TF: Complex regulation of cyp26a1 creates a robust retinoic acid gradient in the zebrafish embryo. PLOS Biol 2007, 5:e304.

58. Piepenburg O, Grimmer D, Williams PH, Smith JC: Activin redux: specification of mesodermal pattern in Xenopus by graded concentrations of endogenous activin B. Development 2004, 131:4977-86.

59. Ramis JM, Collart C, Smith JC: Xnrs and activin regulate distinct genes during Xenopus development: activin regulates cell division. PLOS One 2007, 2:e213.

60. Laping NJ, Grygielko E, Mathur A, Butter S, Bomberger J, Tweed C, Martin W, Fornwald J, Lehr R, Harling J, et al: Inhibition of transforming growth factor (TGF)-beta1-induced extracellular matrix with a novel inhibitor of the TGF-beta type I receptor kinase activity: SB-431542. Mol Pharmacol 2002, 62:58-64.

61. Erter CE, Solnica-Krezel L, Wright CV: Zebrafish nodal-related 2 encodes an early mesendodermal inducer signaling from the extraembryonic yolk syncytial layer. Dev Biol 1998, 204:361-72.

62. Rebagliati MR, Toyama R, Fricke C, Haffter P, Dawid IB: Zebrafish nodalrelated genes are implicated in axial patterning and establishing leftright asymmetry. Dev Biol 1998, 199:261-72.

63. Rebagliati MR, Toyama R, Haffter P, Dawid IB: cyclops encodes a nodalrelated factor involved in midline signaling. Proc Natl Acad Sci USA 1998 95:9932-7.

64. Sampath K, Rubinstein AL, Cheng AM, Liang JO, Fekany K, Solnica-Krezel L, Korzh V, Halpern ME, Wright CV: Induction of the zebrafish ventral brain and floorplate requires cyclops/nodal signalling. Nature 1998, 395:185-9.
65. Cox WG, Hemmati-Brivanlou A: Caudalization of neural fate by tissue recombination and bFGF. Development 1995, 121:4349-58.

66. Pownall ME, Isaacs HV, Slack JM: Two phases of Hox gene regulation during early Xenopus development. Curr Biol 1998, 8:673-6.

67. Mohammadi M, McMahon G, Sun L, Tang C, Hirth P, Yeh BK, Hubbard SR Schlessinger J: Structures of the tyrosine kinase domain of fibroblast growth factor receptor in complex with inhibitors. Science 1997, 276:955-60.

68. Amaya E, Stein PA, Musci TJ, Kirschner MW: FGF signalling in the early specification of mesoderm in Xenopus. Development 1993, 118:477-87.

69. Tsang M, Maegawa S, Kiang A, Habas R, Weinberg E, Dawid IB: A role for MKP3 in axial patterning of the zebrafish embryo. Development 2004, 131:2769-79.

70. Cooke J, Webber JA: Dynamics of the control of body pattern in the development of Xenopus laevis. II. Timing and pattern in the development of single blastomeres (presumptive lateral halves) isolated at the 2-cell stage. J Embryol Exp Morphol 1985, 88:113-33.

71. Woo K, Fraser SE: Specification of the zebrafish nervous system by nonaxial signals. Science 1997, 277:254-7.

72. Shimizu T, Yamanaka Y, Ryu SL, Hashimoto H, Yabe T, Hirata T, Bae YK, Hibi M, Hirano T: Cooperative roles of Bozozok/Dharma and Nodalrelated proteins in the formation of the dorsal organizer in zebrafish. Mech Dev 2000, 91:293-303

73. Sun Z, Jin P, Tian T, Gu Y, Chen YG, Meng A: Activation and roles of ALK4/ ALK7-mediated maternal TGFbeta signals in zebrafish embryo. Biochem Biophys Res Commun 2006, 345:694-703.

74. Gonzalez EM, Fekany-Lee K, Carmany-Rampey A, Erter C, Topczewski J, Wright CV, Solnica-Krezel L: Head and trunk in zebrafish arise via coinhibition of BMP signaling by bozozok and chordino. Genes Dev 2000, 14:3087-92.

75. Lowe CJ, Wu M, Salic A, Evans L, Lander E, Stange-Thomann N, Gruber CE, Gerhart J, Kirschner M: Anteroposterior patterning in hemichordates and the origins of the chordate nervous system. Cell 2003, 113:853-65.

76. Aronowicz J, Lowe CJ: Hox gene expression in the hemichordate Saccoglossus kowalevskii and the evolution of deuterostome nervous systems. Integrative and Comparative Biology 2006, 46:890-901.

77. Lowe CJ, Terasaki M, Wu M, Freeman RM Jr, Runft L, Kwan K, Haigo S, Aronowicz J, Lander E, Gruber C, et al: Dorsoventral patterning in hemichordates: insights into early chordate evolution. PLOS Biol 2006, 4 e291.

78. Nieuwkoop PD: The neural induction process; its morphogenetic aspects. Int J Dev Biol 1999, 43:615-23.

79. Heisenberg CP, Houart C, Take-Uchi M, Rauch GJ, Young N, Coutinho P, Masai I, Caneparo L, Concha ML, Geisler R, et al: A mutation in the Gsk3binding domain of zebrafish Masterblind/Axin1 leads to a fate transformation of telencephalon and eyes to diencephalon. Genes Dev 2001, 15:1427-34.

80. Kim CH, Oda T, Itoh M, Jiang D, Artinger KB, Chandrasekharappa SC, Driever W, Chitnis AB: Repressor activity of Headless/Tcf3 is essential for vertebrate head formation. Nature 2000, 407:913-6.

81. von der Hardt S, Bakkers J, Inbal A, Carvalho L, Solnica-Krezel L, Heisenberg CP, Hammerschmidt M: The Bmp gradient of the zebrafish gastrula guides migrating lateral cells by regulating cell-cell adhesion. Curr Biol 2007, 17:475-87.

82. Niehrs C: On growth and form: a Cartesian coordinate system of Wnt and BMP signaling specifies bilaterian body axes. Development 2010, 137:845-57.

83. Dal-Pra S, Furthauer M, Van-Celst J, Thisse B, Thisse C: Noggin1 and Follistatin-like2 function redundantly to Chordin to antagonize BMP activity. Dev Biol 2006, 298:514-26.

84. Barth KA, Kishimoto Y, Rohr KB, Seydler C, Schulte-Merker S, Wilson SW: Bmp activity establishes a gradient of positional information throughout the entire neural plate. Development 1999, 126:4977-87.

85. Holland ND: Early central nervous system evolution: an era of skin brains? Nat Rev Neurosci 2003, 4:617-27.

86. Marlow HQ, Srivastava M, Matus DQ, Rokhsar D, Martindale MQ: Anatomy and development of the nervous system of Nematostella vectensis, an anthozoan cnidarian. Dev Neurobiol 2009, 69:235-54.

87. Arendt D, Denes AS, Jekely G, Tessmar-Raible K: The evolution of nervous system centralization. Philos Trans R Soc Lond B Biol Sci 2008, 363:1523-8. 
88. Hayward DC, Samuel G, Pontynen PC, Catmull J, Saint R, Miller DJ, Ball EE: Localized expression of a dpp/BMP2/4 ortholog in a coral embryo. Proc Natl Acad Sci USA 2002, 99:8106-11.

89. Finnerty JR, Pang K, Burton P, Paulson D, Martindale MQ: Origins of bilateral symmetry: Hox and dpp expression in a sea anemone. Science 2004, 304:1335-7.

90. Matus DQ, Pang K, Marlow H, Dunn CW, Thomsen GH, Martindale MQ: Molecular evidence for deep evolutionary roots of bilaterality in animal development. Proc Natl Acad Sci USA 2006, 103:11195-200.

91. Rentzsch F, Anton R, Saina M, Hammerschmidt M, Holstein TW, Technau U: Asymmetric expression of the BMP antagonists chordin and gremlin in the sea anemone Nematostella vectensis: implications for the evolution of axial patterning. Dev Biol 2006, 296:375-87.

92. Saina M, Genikhovich G, Renfer E, Technau U: BMPs and chordin regulate patterning of the directive axis in a sea anemone. Proc Natl Acad Sci USA 2009, 106:18592-7.

93. Reversade B, De Robertis EM: Regulation of ADMP and BMP2/4/7 at opposite embryonic poles generates a self-regulating morphogenetic field. Cell 2005, 123:1147-60.

94. Ben-Zvi D, Shilo BZ, Fainsod A, Barkai N: Scaling of the BMP activation gradient in Xenopus embryos. Nature 2008, 453:1205-11.

95. Lapraz F, Besnardeau L, Lepage T: Patterning of the Dorso-Ventral Axis in Echinoderms: Insights into the Evolution of BMP-Chordin Signaling Network. PLOS Biol 2009, 7:e1000248.

96. Gerhart J, Lowe C, Kirschner M: Hemichordates and the origin of chordates. Curr Opin Genet Dev 2005, 15:461-7.

97. Nomaksteinsky M, Rottinger E, Dufour HD, Chettouh Z, Lowe CJ, Martindale MQ, Brunet JF: Centralization of the deuterostome nervous system predates chordates. Curr Biol 2009, 19:1264-9.

98. De Robertis EM: Evo-devo: variations on ancestral themes. Cell 2008, 132:185-95.

99. Yu JK, Satou Y, Holland ND, Shin IT, Kohara Y, Satoh N, Bronner-Fraser M, Holland LZ: Axial patterning in cephalochordates and the evolution of the organizer. Nature 2007, 445:613-7.

100. Onai T, Lin HC, Schubert M, Koop D, Osborne PW, Alvarez S, Alvarez R, Holland ND, Holland LZ: Retinoic acid and Wnt/beta-catenin have complementary roles in anterior/posterior patterning embryos of the basal chordate amphioxus. Dev Biol 2009, 332:223-33.

101. Petersen $C P$, Reddien PW: Wnt signaling and the polarity of the primary body axis. Cell 2009, 139:1056-68.

102. Martindale MQ: The evolution of metazoan axial properties. Nat Rev Genet 2005, 6:917-27.

103. Wikramanayake $A H$, Hong $M$, Lee PN, Pang K, Byrum CA, Bince JM, Xu R, Martindale MQ: An ancient role for nuclear beta-catenin in the evolution of axial polarity and germ layer segregation. Nature 2003, 426:446-50.

104. Lee PN, Pang K, Matus DQ, Martindale MQ: A WNT of things to come: evolution of Wnt signaling and polarity in cnidarians. Semin Cell Dev Biol 2006, 17:157-67.

105. Westerfield M: The zebrafish book: a guide for the laboratory use of zebrafish (Brachydanio rerio). Eugene, OR: M. Westerfield; 1993.

106. Feldman B, Stemple DL: Morpholino phenocopies of sqt, oep, and ntl mutations. Genesis 2001, 30:175-7.

107. Karlen S, Rebagliati M: A morpholino phenocopy of the cyclops mutation. Genesis 2001, 30:126-8.

108. Thisse C, Thisse B: Antivin, a novel and divergent member of the TGFbeta superfamily, negatively regulates mesoderm induction. Development 1999, 126:229-40.

109. Morita T, Nitta H, Kiyama Y, Mori H, Mishina M: Differential expression of two zebrafish emx homeoprotein mRNAs in the developing brain. Neurosci Lett 1995, 198:131-4.

110. Prince VE, Joly L, Ekker M, Ho RK: Zebrafish hox genes: genomic organization and modified colinear expression patterns in the trunk. Development 1998, 125:407-20.

111. Miller-Bertoglio VE, Fisher S, Sanchez A, Mullins MC, Halpern ME: Differential regulation of chordin expression domains in mutant zebrafish. Dev Biol 1997, 192:537-50.

doi:10.1186/1471-213X-11-26

Cite this article as: Varga et al:: Correct anteroposterior patterning of the zebrafish neurectoderm in the absence of the early dorsal organizer. BMC Developmental Biology 2011 11:26.

\section{Submit your next manuscript to BioMed Central and take full advantage of:}

- Convenient online submission

- Thorough peer review

- No space constraints or color figure charges

- Immediate publication on acceptance

- Inclusion in PubMed, CAS, Scopus and Google Scholar

- Research which is freely available for redistribution 